\title{
A Cross-Layer Packet Scheduling Scheme for Multimedia Broadcasting via Satellite Digital Multimedia Broadcasting System
}

\author{
Hongfei Du, Linghang Fan, Upendra Mudugamuwa, and Barry G. Evans, University of Surrey
}

\begin{abstract}
In recent years, multimedia content broadcasting via satellite has attracted increased attention. The satellite digital multimedia broadcasting (S-DMB) system has emerged as one of the most promising alternatives for the efficient delivery of multimedia broadcast multicast service (MBMS). The design of an efficient radio resource management (RRM) strategy, especially the packet scheduling scheme, becomes a key technique for provisioning multimedia services at required quality of service (QoS) in S-DMB. In this article, we propose a novel cross-layer packet scheduling scheme that consists of a combined delay and rate differentiation (CDRD) service prioritization algorithm and a dynamic rate matching (DRM)-based resource allocation algorithm. The proposed scheme considers multiple key factors that span from the application layer to the physical layer, aiming at simultaneously guaranteeing diverse QoS while utilizing radio resources efficiently under the system power and resource constraints. Simulation results demonstrate that the proposed cross-layer scheme achieves significantly better performance than existing schemes in queuing delay, jitter, and channel utilization.
\end{abstract}

\section{INTRODUCTION}

Recent advances in the digital multimedia broadcasting (DMB) industry have offered the network operator a platform to deliver multimedia services to the mass market in a spectrum-efficient and cost-effective way. A variety of initiatives, such as multimedia broadcast and multicast services (MBMS), digital video broadcastinghandheld (DVB-H), and terrestrial/satellite-digital multimedia broadcasting (T/S-DMB) are envisaged to provide one-to-many content distribution to mobile users. The 3rd Generation Partnership Project (3GPP) MBMS framework [1] defines a unidirectional point-to-multipoint mode for providing multimedia services and thereby, optimizes the available capacity in cellular networks. DVB-H [2], as initiated by the DVB forum, implements additional features based on the DVB-T standard to address the specific constraints associated with mobile handheld terminals. At the same time, the media forward link only (MediaFLO) [3] air interface specification developed by Qualcomm was recently approved by the Telecommunications Industry Association (TIA) as a new air interface standard for multicast delivery, aimed at delivering cost-efficient and high-quality multimedia services to the U.S. mobile market.

As a complementary alternative to $3 \mathrm{G}$ mobile networks, the S-DMB system is attracting a great deal of attention within the satellite community [4] as a cost-effective approach for the delivery of MBMS to mobile users over satellite broadcasting networks. Based on its broadcast nature, the S-DMB system offers extensive coverage, low transmission cost for large numbers of terminals, as well as high QoS guarantees for multimedia provisioning. By employing the wideband code-division multiple access (WCDMA) with frequency division duplexing (FDD), the system can be closely integrated with existing mobile cellular networks and minimize the potential cost impact on both $3 \mathrm{G}$ cellular terminals and network operators. The whole range of issues pertinent to the S-DMB system, from system definition to demonstration and validation, are addressed in the following European IST projects: Mobile Applications \& sErvices Satellite \& Terrestrial inteRwOrking (MAESTRO) and Mobile Digital broadcast Satellite (MoDiS).

Given the unidirectional nature and the pointto-multipoint services it provides that are aimed at maximizing spectrum efficiency and satisfying diverse QoS, the design of radio resource management (RRM) functionality implemented at the S-DMB access layer is challenging. The packet scheduling, which is the single function performing short-term resource allocation, is the focus of efficient resource allocation.

Herein the packet scheduling is envisaged at 


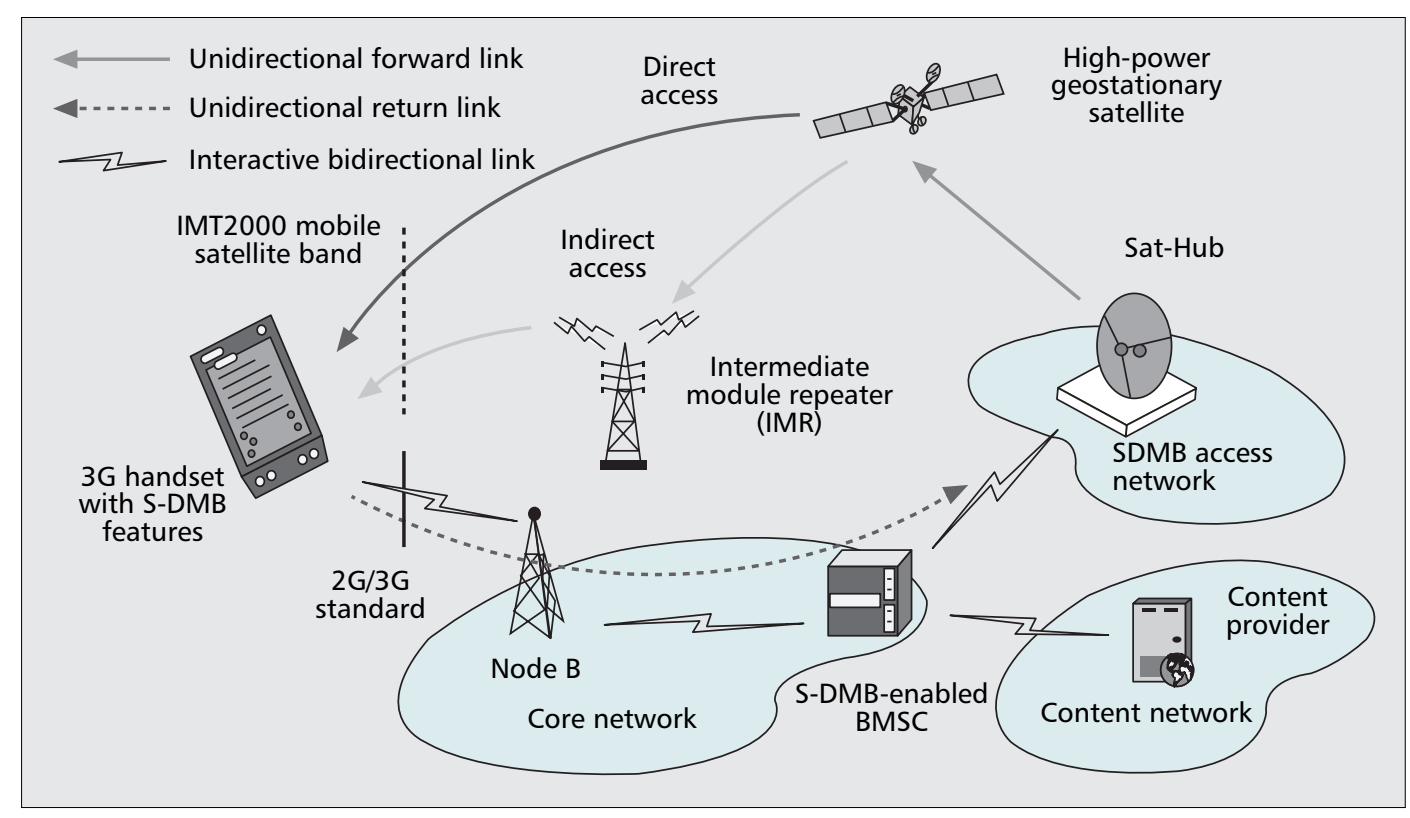

The S-DMB radio
interface employs an
adaptation of the
WCDMA, with the
satellite gateway
hosting both the
radio network
controller and the
Node B functional
entities. The user
equipment applies
the standard $3 G$
terminal, enriched
with S-DMB-enabling
functions.

Figure 1. S-DMB concept overview and system architecture.

the medium access control (MAC) layer for multisessions with diverse QoS requirements, where each session employs the proposed dynamic rate matching (DRM) technique at the physical layer. The proposed scheme considers multiple crosslayer criteria to achieve both efficiency and fairness. First, the service prioritization procedure takes into account the service types and QoS requirements in terms of maximum tolerable queuing delay and required data rate. Second, the queuing dynamics are envisaged effectively into service prioritization to track the instantaneous traffic variations at the radio link control (RLC) layer. Furthermore, the instantaneous data rate at the transport channel ( $\mathrm{TrCH})$ is envisaged as another important criterion to minimize the unnecessary discontinuous transmission (DTX) and thereby, optimize the resource utilization.

The proposed cross-layer packet scheduling algorithm is distinct from most existing schemes $[5,6]$ in that:

- It guarantees the prescribed QoS requirements.

- Simultaneously, it considers multiple crosslayer criteria and balances the priority and fairness.

- A DRM technique is applied to resource allocation to reduce unnecessary puncturing and repetition and optimize the transmit power.

To the best of our knowledge, this is the first work to investigate the cross-layer optimization that combines QoS requirements, queuing dynamics, and rate matching information together in the packet scheduling decision.

The article continues with the background description of the S-DMB system and the respective issues pertinent to RRM, highlighting some research challenges and opportunities for packet scheduling design. The subsequent section details the proposed cross-layer packet scheduling scheme. Simulation performance of the proposed scheme is then presented, followed by the conclusion of the article.

\section{BACKGROUND}

\section{S-DMB SYSTEM ARCHITECTURE}

As illustrated in Fig. 1, the S-DMB system defines a hybrid satellite-terrestrial communication system, featuring a geostationary satellite component that is responsible for MBMS delivery and provides a European coverage by multiple umbrella cells. Being closely integrated into the $2.5 \mathrm{G} / 3 \mathrm{G}$ baseline architecture, the system enjoys maximum reuse of technology and infrastructure and minimum system development cost. The hybrid system takes advantage of the satellite broadcast capability to provide efficient delivery of MBMS content to an extensive mass mobile market.

The S-DMB radio interface employs an adaptation of the WCDMA, with the satellite gateway hosting both the radio network controller (RNC) and the Node B functional entities. The user equipment (UE) applies the standard $3 \mathrm{G}$ terminal, enriched with S-DMB-enabling functions, which given the unidirectional nature, are very limited. The terrestrial gap-fillers, identified as intermediate module repeater (IMR), are collocated at the terrestrial base stations to enhance signal reception quality and provide adequate coverage in urban and built-up areas. The SDMB-enabled broadcast/multicast service center (BMSC), is enhanced with S-DMB-specific functions from the standard 3GPP MBMS BMSC. It is noteworthy that no direct return link via satellite is envisaged under the baseline S-DMB infrastructure; rather, the return path is provided via the terrestrial networks if required.

\section{RRM}

In S-DMB, the RRM functionality comprises three main parts: radio resource allocation (RRA), packet scheduling, and admission control. This functionality cooperates interactively during resource allocation procedures.

The RRA is responsible for the radio bearer configuration at the beginning of each session start, 
The provisioning of

multimedia

applications involves

highly variable bit

rates. Therefore, it is

highly desirable that

the available radio

resources can be

utilized efficiently

and adaptively in

response to the data

rate dynamics. which includes the estimation of the number of required logical/transport/physical channels including their mappings for each physical channel.

The packer scheduling operates periodically in each transmission time interval (TTI) of the radio bearers, performing the time-multiplexing and power allocation tasks on the basis of service $\mathrm{QoS}$ requirements and system constraints.

The admittance decision of each incoming MBMS session is handled by the admission control during the phase of service establish/renegotiation, aiming at preserving the required QoS and achieving efficient resource allocation.

\section{PACKet SCHEDULING}

The packet scheduling in the S-DMB system is responsible for two main tasks:

- It time-multiplexes competing flows with diverse QoS into physical channels, aimed at satisfying the service QoS requirements.

- It adjusts the transmit powers for the physical channels on the basis of the packet size to be served and the required reception quality of the service, in terms of the target block error rate (BLER).

Given the absence of real-time feedback information from UE, the impact of the packet scheduler on the overall system performance is limited. However, its role in assuring comprehensive QoS guarantees remains crucial for multimedia provisioning.

\section{Rate Matching}

The provisioning of multimedia applications involves highly variable bit rates. Therefore, it is highly desirable that the available radio resources can be utilized efficiently and adaptively in response to the data rate dynamics.

To satisfy the physical layer supportable frame structure prior to the transport channel multiplexing, rate matching (RM) is performed at the physical layer to match the encoded $\mathrm{TrCH}$ data rates to the allowed physical channel data rates by either puncturing or repeating bits to the $\operatorname{TrCH}$. The objective of this function is to minimize unnecessary DTX insertions so as to reduce the transmission power-off periods.

Time multiplexing from $\operatorname{TrCH}$ to physical channels is performed independently from other physical layer functions, such as CRC addition, turbo coding, and interleaving. However, the RM function, which is an essential part of the transport channel coding and multiplexing mechanism, plays a crucial role in efficient resource allocation.

\section{Motivations and Research Challenge}

The unique features of the S-DMB system restrict the effectiveness and efficiency on shortterm resource allocation functions. The design of packet scheduling proves to be challenging for the following reasons:

- The system suffers from the long delay associated with the geostationary satellite link, which makes the channel-state-dependent scheduling and the fast power control mechanism unfeasible.

- Its unidirectional nature accompanied with the built-in broadcast multicast mode further aggravates the design difficulty on a feasible packet scheduling scheme.
- The total available power for all the physical channels within a single satellite beam is limited, and the packet scheduler must be designed such that it satisfies the power constraint and minimizes unnecessary power consumption.

- Multimedia applications feature stringent and diverse QoS requirements, hence the design of a packet scheduler must take into account both the differentiation and fulfillment of these requirements.

Therefore, the design of an efficient packet scheduling scheme in S-DMB requires comprehensive considerations on both system constraints and QoS satisfactions.

Although a variety of packet scheduling algorithms are proposed in the literature for both wired and wireless networks [5], they cannot be applied directly to the S-DMB network due to its unique architectural constraints. Previous studies [6] have systematically addressed the packet scheduling problems in S-DMB via adaptation of two wellknown scheduling schemes, namely multi-level priority queuing (MLPQ) and weighted fair queuing (WFQ), both of which feature major weaknesses in providing QoS-differentiated multimedia services with respect to efficiency and fairness.

MLPQ serves a queue, based on its traffic priority; low-priority service may suffer from considerably longer queuing delays. Round-robin is employed among queues with the same priority, providing no differentiation for services with the same QoS rank.

In WFQ, queues are prioritized based on their required data rates, while the time-stamps of the head packets are considered for queues with the same data rate. Since no QoS differentiation is envisaged, the performance of WFQ is even worse than that of MLPQ in terms of both delay and delay variation (jitter) [6].

An existing static rate matching (SRM) technique calculates the rate matching ratios (RMR), that is, the percentage of bits required to be punctured/repeated against the total information data, based on the maximum allowed data rates of the $\operatorname{TrCH}$ at the beginning of each session start. RMR remain unchanged during the session transmission. Consequently, unnecessary DTX insertion in the physical layer occurs whenever the instantaneous data rates of $\mathrm{TrCH}$ are less than their maximum data rates. This can result in unnecessary puncturing and repetition and inefficient resource and power utilization because the base station transmit power is wasted during these DTX power-off periods.

\section{Cross-Layer Packet Scheduling \\ OVERVIEW}

Figure 2 illustrates the layer/sublayer interactions of the proposed cross-layer packet scheduling scheme. The RRM mainly is handled at the data link layer, which can be further divided into radio resource control (RRC), RLC, and MAC sublayers. The proposed cross-layer/sublayer correspondence is set up, from both upward and downward directions. During the radio bearer configuration, the RRA abstracts the prescribed QoS demands from each session when it starts and passes them to the packet scheduler as one set of priority cri- 


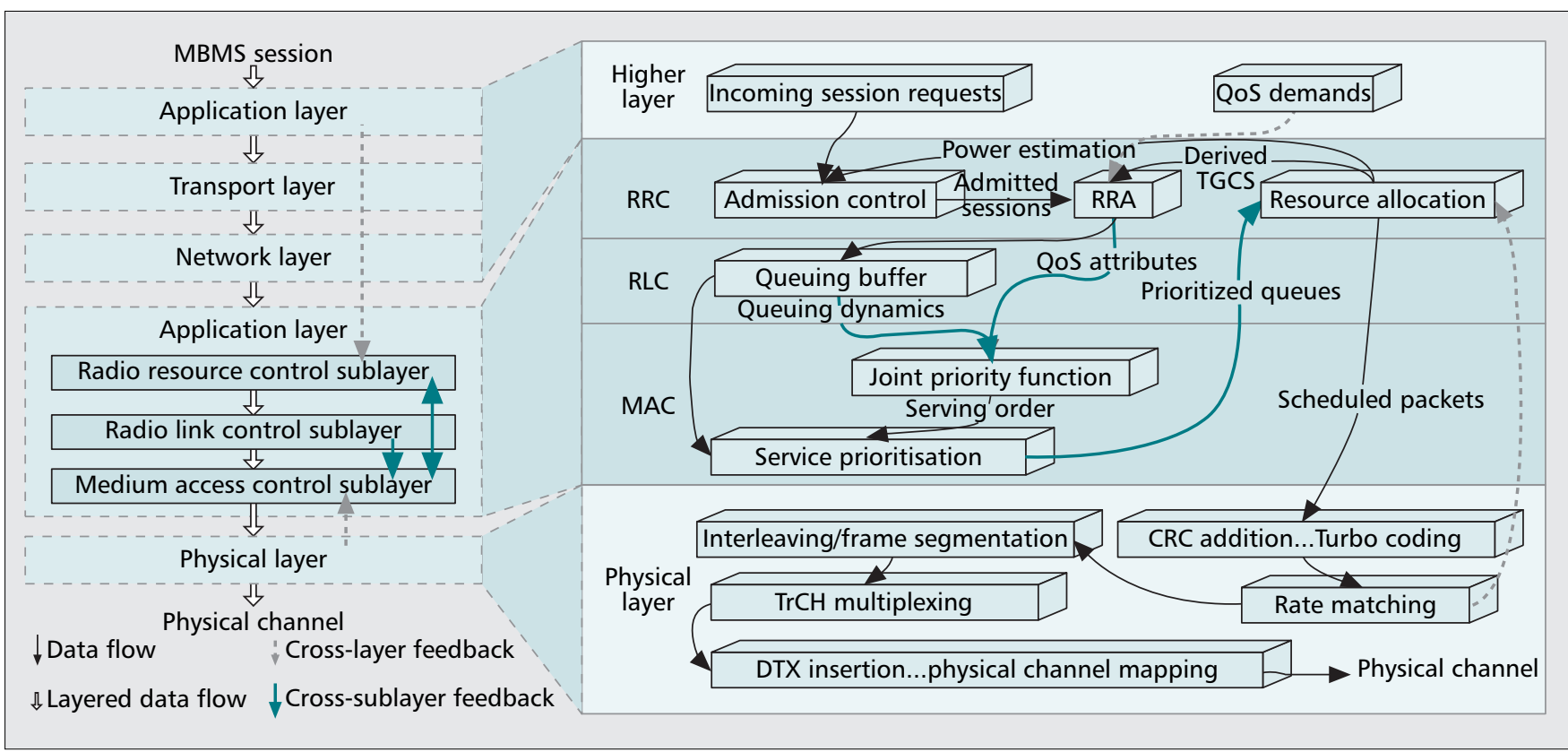

Figure 2. Illustration of the layer interactions of the proposed cross-layer packet scheduling scheme.

teria. The queuing dynamics in the RLC buffer are monitored and passed to the packet scheduler as another set of priority criteria. We employ a joint priority function (JPF) in the packet scheduler to handle the previous priority criteria and derive the serving orders of competing flows.

The parameters considered in the JPF can be grouped into two categories:

- QoS parameters

- Queuing dynamic parameters

The parameters in the first category depend on the application and are derived on a per-session scale that remains constant during the session transmission, while the parameters in the second category track the instantaneous queuing behaviors at the forward access channel (FACH) queue in the RLC buffer on a per-TTI scale.

In the S-DMB system, the service types are considered as streaming, hot download, and cold download and described as follows:

- Streaming enables multimedia to be stored temporarily in the receiver buffer and displayed continuously even before the completion of transmission. Service in this category requires explicit upper bound on queuing delay and jitter.

- Hot download enables the data to be stored at the receiver for offline access. Compared with streaming, the hot download service has more tolerant demand on delay and jitter but more stringent demand on packet loss.

- Cold download requires the least demand on delay and jitter but the most stringent demand on packet loss. Services in this category are often transmitted as an individual file, such as software packages, video/images, and text messages.

There is a one-to-one correspondence between an MBMS session and an MBMS pointto-multipoint traffic channel (MTCH) logical channel. The logical channels then are mapped in a one-to-one manner to the FACH transport channel. The scheduled packets are delivered to the secondary common control physical channel (S-CCPCH) in the form of transport block (TB) [7]. One or more FACH(s) are carried by a single S-CCPCH via transport channel multiplexing at the physical layer. For each active physical channel, the exact format of the transport format combination (TFC), which consists of multiple transport block sets (TBS), is selected from the transport format combination set (TFCS).

The framework of the proposed packet scheduling scheme is illustrated in Fig. 3. The packet scheduling strategy can be conceptualized into the following two main steps:

1. Service prioritization: The incoming service requests are ordered according to the priority criteria. In selecting the respective criteria, the service attributes are considered to provide the scheduling task while considering multiple and essential QoS factors.

2. Resource allocation: After all of the multiplexed sessions are prioritized, resources are allocated accordingly to these sessions that consist of bit rate and transmit power assignments within the specific resource allocation interval (i.e., one TTI).

\section{CDRD Service Prioritization}

Advances in multimedia applications require the RRM scheme to support diverse QoS among heterogeneous traffic. The proposed CDRD service prioritization algorithm takes into account multiple key criteria simultaneously for assuring comprehensive QoS satisfaction. On the one hand, the CDRD scheme considers the application prescribed QoS requirements as a combination of multiple attributes including traffic priority, required data rate, and queuing delay constraint. On the other hand, the queuing dynamics of the competing flows at the RLC layer are captured for further scheduling optimization.

As illustrated in Fig. 3, each session is assumed to retain an individual FACH queue in the RLC 


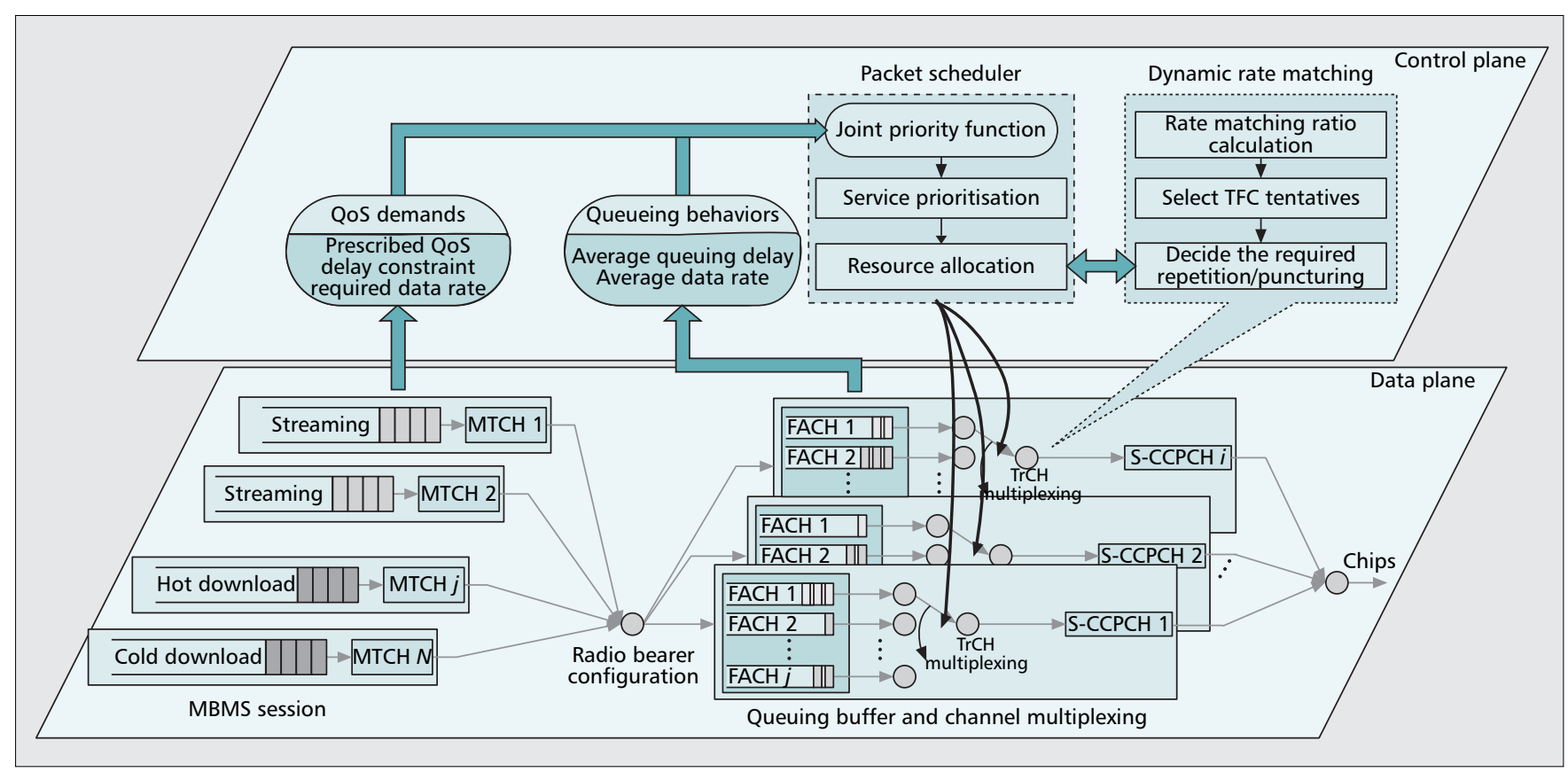

Figure 3. The proposed packet scheduling procedure implemented at the S-DMB hub.

buffer. Packets in the FACH queues are prioritized according to their respective performance metrics. The packet scheduler handles all the FACH queues according to their instant joint priorities rather than the service types of the carried traffic. By considering multiple performance criteria, the packet scheduler can balance the performance in various aspects among heterogeneous multimedia traffic with diverse QoS preferences.

In CDRD, the following criteria in the JPF are considered:

- QoS class factor - This factor is essentially a time-independent parameter designated for each queue, representing heterogeneous service types amongst competing sessions.

- Data rate factor - This factor is calculated as the ratio of the required data rate against the average transmitted data rate until current TTI. This factor effectively drives the instantaneous achieved data rate of the service to its required data rate.

- Delay constraint factor - This attribute is determined according to the difference between the average queuing delay and the maximum queuing delay tolerated by the corresponding service (i.e., delay threshold). Delay threshold can be regarded as a tunable parameter upon balancing the system performance. This factor is only effective when the average queuing delay is beyond the designated delay threshold.

In each TTI, the scheduler sorts the FACH queues in descending order, according to their instantaneous priorities calculated from the JPF function. Subsequently, the FACH queues with higher priorities are served prior to their counterparts with lower priority.

From the implementation point of view, the proposed CDRD algorithm introduces extra computation complexity due to its nonlinear (with loop iterations for selection/sort operation) and nondeterministic (with unpredictable variables) nature.
To examine the scalability of the proposed CDRD algorithm, the Big O notation [8] is employed for determining the involved computational complexity. We assume there are $n$ sessions to be transmitted to the UE that are located in multiple sectors of a satellite beam. The computational complexity for CDRD algorithm is computed for a single TTI period. With the input size of the total number of the FACH denoted by $n$, the CDRD algorithm requires an overall computational complexity of $O\left(n^{2}\right)$, featuring typical quadratic statistics.

\section{Dynamic Resource Allocation}

Dynamic Rate Matching - To minimize the DTX insertion and optimize the total power consumption in downlink SRM, the DRM technique was proposed for S-DMB in [9]. The objective of downlink DRM is to minimize the number of DTX bits required for the selected TFC at a given TTI according to the available physical layer resources. Rather than the rate matching calculation in SRM, in the DRM, the RMR is calculated in each TTI, based on the instantaneous data rate of each $\mathrm{TrCH}$. Therefore, the DRM employs a variable RMR to prevent unnecessary DTX insertions. In each TTI, the following phases are performed in DRM:

- Phase 1 (TFC reordering): all the TFC within the TFCS list are re-ordered according to their corresponding total data rates.

- Phase 2 (RMR calculation): the RMR is calculated, based on the instantaneous data rate (i.e., the TBS size) of each $\mathrm{TrCH}$ for each different TFC allowed for a given physical channel.

- Phase 3 (bit matching): according to the selected TFC, a tentative value for repetition and/or puncturing bits is calculated for each $\mathrm{TrCH}$.

Finally, the rate matching module performs the tentative value corrections, and a rate matching pattern is generated. 
To apply the DRM technique, modifications are identified and applied at both the sending and receiving side as follows:

- A new rate matching interval in TTI-scale is identified and applied, during which RMR remains constant.

- Before the transmission starts, RMR must be calculated for all possible TFC.

Note that the DRM can facilitate higher bit repetition, which has an essential impact on improving the bit error rate performance. The transmit power that was saved can be utilized for better performance on transmission capacity or packet loss rate. The main limitation of DRM is that more processing and memory are required for the rate matching calculation and storage.

DRM-based Resource Allocation - The task of resource allocation is to select the required bit rate and transmit power for all physical channels within the specific resource allocation interval (i.e., one TTI), subject to system constraints. An existing SRM technique at the physical layer has been traditionally designed separately from higher layers. Based on the novel DRM technique at the physical layer, we develop a crosslayer design that optimizes the resource utilization at the data link layer to improve the overall system efficiency when combined with resource allocation at the data link layer.

Unlike SRM, where the RMR is fixed during the session transmission, in DRM, until all the sessions are scheduled, the precise RMR for each $\mathrm{TrCH}$ is unknown, and the DRM only knows the possible TFC candidates (i.e., a TFC subset). The power requirements for $\mathrm{TrCH}$ are evaluated based on every possible TFC within the TFC subset. In each TTI, a TFC is selected for the given physical channel; the DRM then calculates the number of bits required to be repeated or punctured.

The proposed DRA algorithm, as shown in Fig. 4, relies on the DRM technique to evaluate the required transmit power for respective $\mathrm{TrCH}$ according to their instantaneous data rate requirements. In this case, the physical layer instantaneous supportable data rate is effectively the TBS size that is allowed for the total transmit power estimation. In addition to the priority decided by the service prioritization function, the instantaneous data rate information is fed in to the resource allocation module for the power estimation. The derived TFC is thereby priority-based, DRM-associated, and data-rateconfined, which is then allocated to the corresponding physical channel. In conclusion, the DRA algorithm offers two main advantages:

- It allows better DTX minimization.

- It requires less transmit power when the instantaneous data rates are less than the maximum data rate.

\section{Performance Evaluation}

\section{SimULATION SCENARIOS}

To demonstrate the performance enhancement of the proposed cross-layer packet scheduling scheme, a system-level simulator implementing the S-DMB system was developed with the aid of the software package ns-2. The packet scheduler is physically implemented in the satellite gateway.

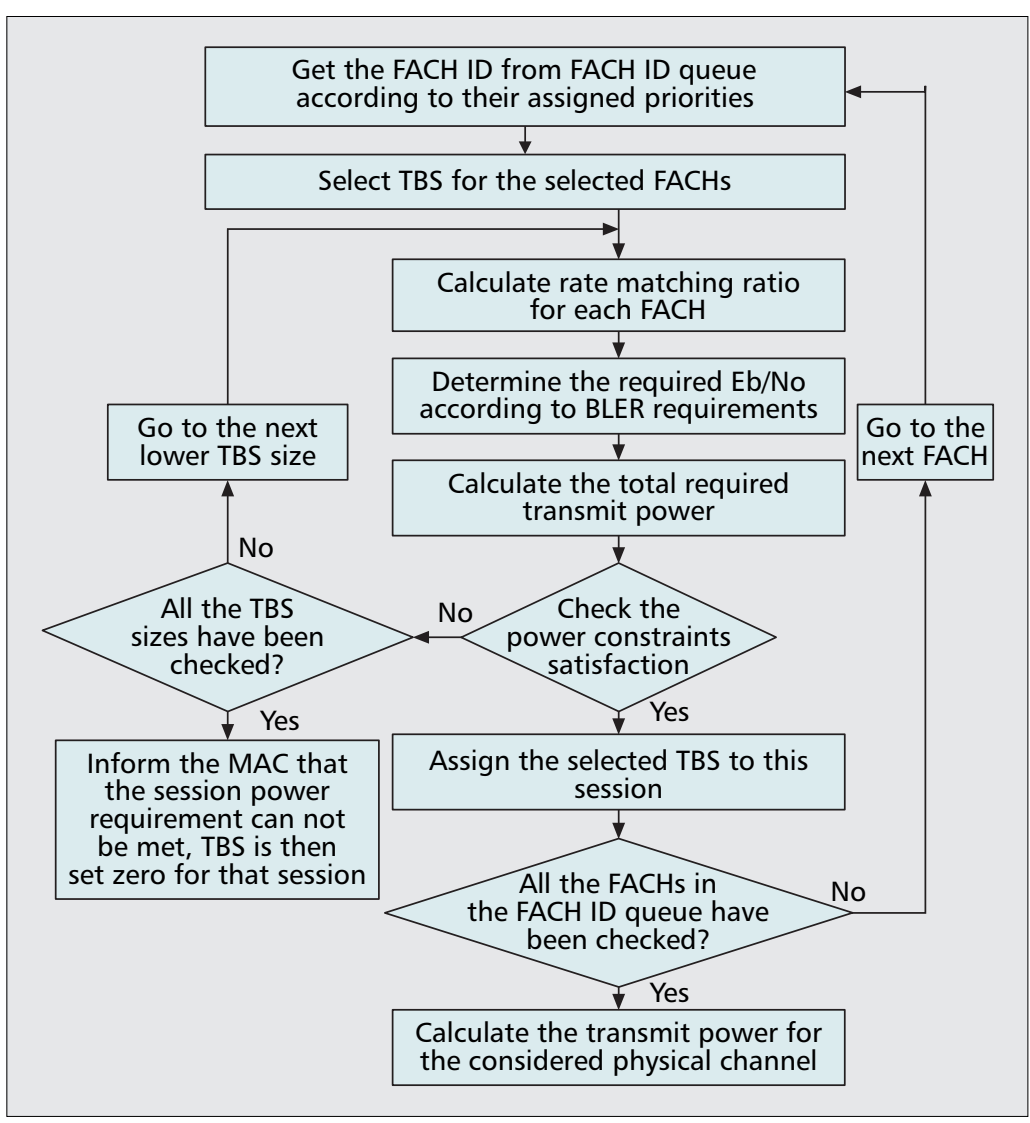

Figure 4. Flowchart for the DRA algorithm.

The streaming traffic model applies publicly available trace files for video streaming traffic. Traffic characteristics associated with hot and cold download services follow the Pareto distribution. In addition, we examine the performance between users with different data rates. Due to the space limitation, an indicative and persuasive scenario is selected to discuss the simulation outcomes as:

- S-CCPCH 1 carries streaming FACH 2 and 3 with data rates of $256 \mathrm{~kb} / \mathrm{s}$ and $64 \mathrm{~kb} / \mathrm{s}$, respectively, and hot download $\mathrm{FACH} 1$ with a data rate of $64 \mathrm{~kb} / \mathrm{s}$.

- S-CCPCH 2 carries streaming FACH 4 and 5 with data rates of $256 \mathrm{~kb} / \mathrm{s}$ and $128 \mathrm{~kb} / \mathrm{s}$, respectively.

- S-CCPCH 3 carries cold download FACH 6 with a data rate of $384 \mathrm{~kb} / \mathrm{s}$.

\section{System Performance Results}

Queuing Delay Evaluation - First, the mean queuing delay experienced by packets in each FACH queue at the RLC buffer is investigated. As illustrated in Fig. 5, downloaded multimedia services experience much less mean queuing delay in CDRD than in MLPQ, while the mean delays experienced by streaming services feature similar performance. It can be inferred that the significant reduction on the delay of the lower QoS class does not pose significant performance degradation on its higher QoS class counterpart, that is, the QoS of streaming can be guaranteed. The cumulative distribution function (CDF) of queuing delays for the respective streaming FACH verifies that CDRD achieved better queuing delay distribution than MLPQ for all the streaming FACH. 


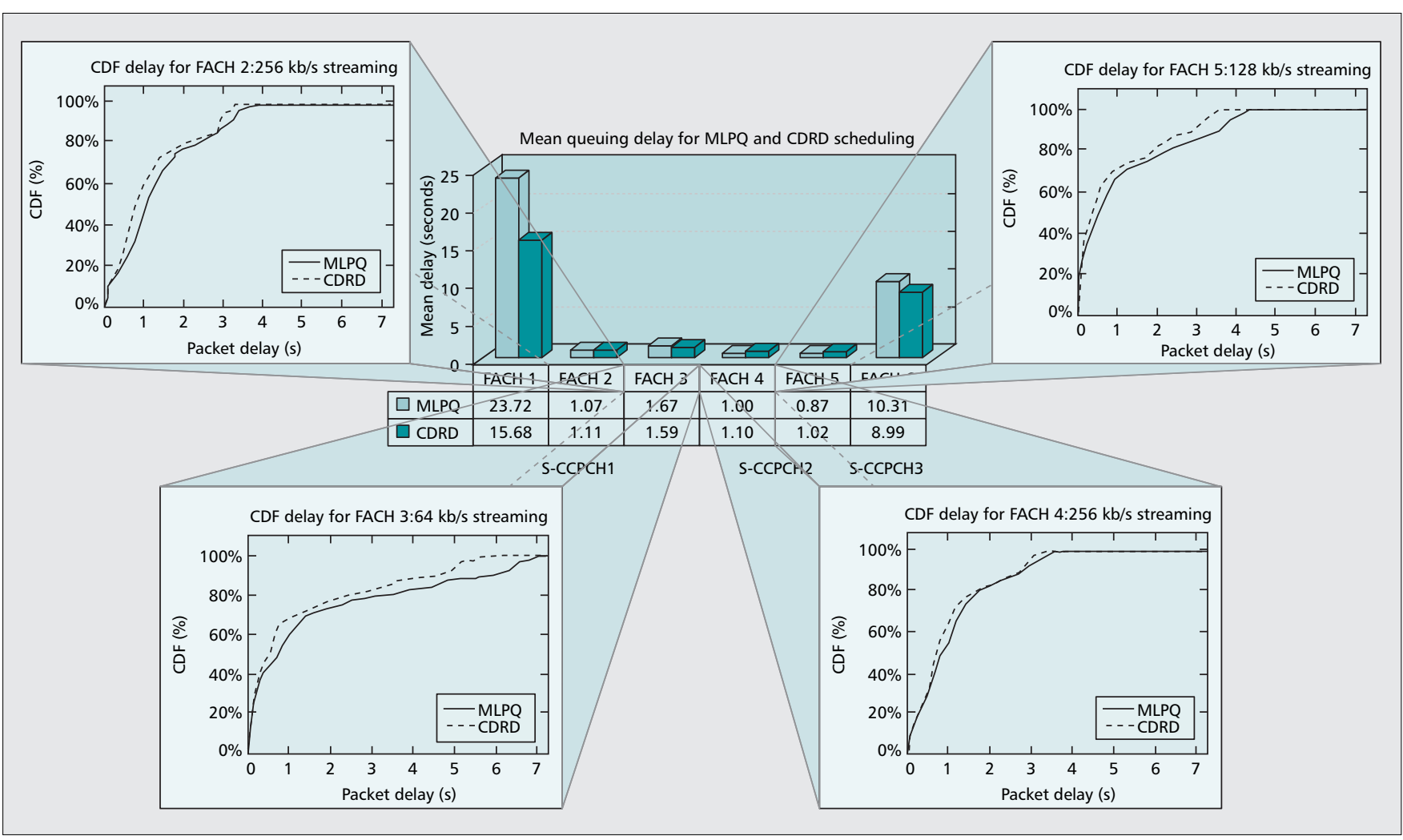

Figure 5. Queuing delay performance and CDF distribution for streaming services.

It is worth noting that the streaming service in S-DMB is quite sensitive to delay variation, thereby the queuing jitter of the flows should be limited to preserve the time variation between packets in the stream [10]. The simulation results prove that the CDRD achieves much lower queuing jitter for both streaming and download services, which makes it an attractive approach for delivering jitter-sensitive multimedia services.

As mentioned previously, the delay threshold is an adjustable parameter upon balancing the system performance. Simulation results prove that a more stringent delay threshold leads to better performance for the corresponding QoS traffic class and causes longer delays for the others. It is worth mentioning that the JPF function employs multiple performance criteria for determining the traffic serving priority that effectively prevents queues with performance degradation on a single profile from gaining extra unnecessary resources. In the case that a flow experiences instantaneous extra burst traffic, the increase on its queue length may lead to its queuing delay out of profile. However, extra incoming traffic can also lead to instantaneous increase on the transmitted data rate, which effectively influences its serving priority and prevents the queue from obtaining unnecessary additional resources.

Physical Channel Utilization Evaluation Figure 6 depicts the instantaneous physical channel utilization achieved for different scheduling and rate matching schemes during a sample simulation period. On the one hand, the total channel utilization achieved by CDRD enjoys remarkable improvement, compared with MLPQ. On the other hand, by effectively utilizing wasted resources via minimizing the unnecessary puncturing and repetition, the DRM-based resource allocation outperforms its SRM-based counterpart with a significant performance gap.

It is noteworthy that the heterogeneity of traffic mixture in an S-CCPCH can have an essential impact on the physical channel utilization. It is shown that the performance gain in the S-CCPCH channel utilization is higher in a more heterogeneous traffic mix scenario (e.g., S-CCPCH 1) than those in other relatively simple scenarios (e.g., S$\mathrm{CCPCH} 2$ and 3 ). Therefore, this proves that the proposed algorithm is capable of offering a higher resource utilization score when the traffic mix in the S-CCPCH becomes more heterogeneous.

Note that the impact of TTI variation on the performance of the proposed scheduling mechanism is two-fold. On the one hand, the simulation results proved that a higher TTI setting improves the system performance on the throughput and channel utilization. On the other hand, we found that a lower TTI setting is capable of providing higher sensitivity for capturing the traffic dynamics.

\section{CONCLUSION}

In this article, we propose a cross-layer packet scheduling scheme for multimedia delivery in the S-DMB system. This scheme not only takes into account the impact of key performance factors reflecting service QoS demands and queuing dynamics, but also utilizes a DRM technique at the physical layer to maximize spectrum efficiency and resource utilization. Simulation was conducted over extensive scenarios for various performance metrics. Results show that the proposed packet scheduling scheme achieves better 


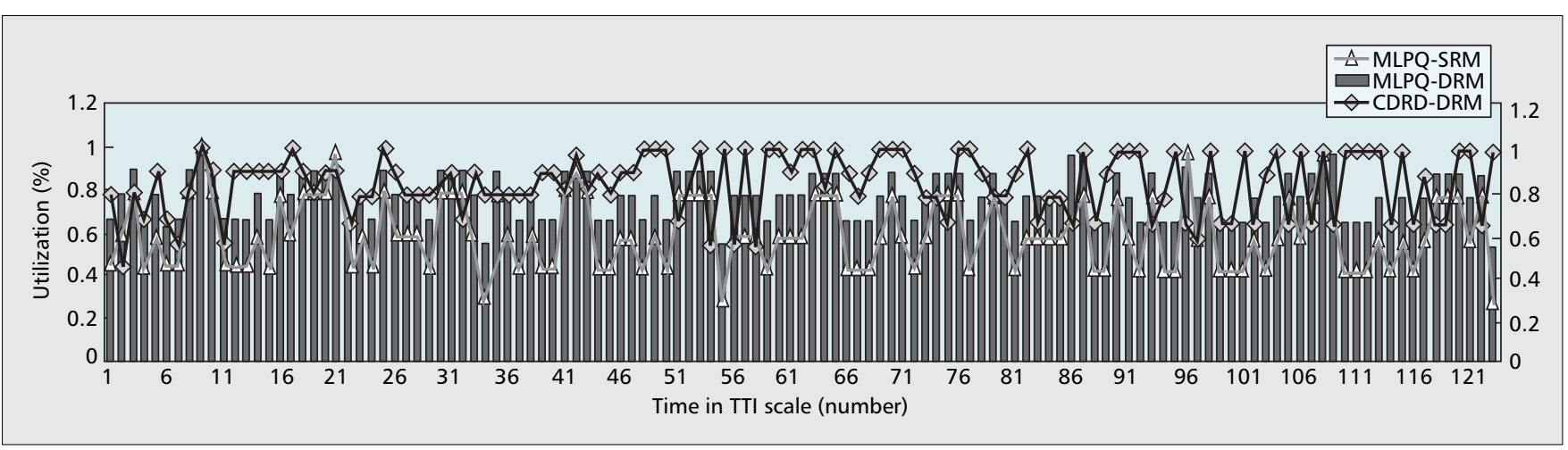

Figure 6. Instantaneous physical channel utilization for MLPQ and CDRD scheduling using SRM and DRM techniques.

performance than the existing schemes regarding delay, jitter, and channel utilization. Discussions on the impact of threshold parameter and traffic heterogeneity on system performance further demonstrate the flexibility and scalability that can be achieved in this scheme.

\section{ACKNOWLEDGMENTS}

This work was supported by the European Commission (EC) within the context of the IST FP6 MAESTRO and Satellite communications Network of Excellence (SatNEx) projects. The financial contribution of the EC towards these projects is greatly appreciated. The authors would like to thank Dr. Atta Quddus for his valuable discussion on physical layer rate matching. The authors also would like to thank all the anonymous reviewers for their constructive comments that improved the presentation of this article.

\section{REFERENCES}

[1] 3GPP TS 23.246 v6.8.0, "Multimedia Broadcast/Multicast Service; Architecture and Functional Description," Rel. 6, 2 Oct. 2005

[2] ETSI EN 302 304, "Digital Video Broadcasting: Transmission System for Handheld Terminals (DVB-H)," Nov. 2004.

[3] TIA-1099, "Forward Link Only Air Interface Specification for Terrestrial Mobile Multimedia Multicast," Sept. 2006.

[4] N. Chuberre et al., "Relative Positioning of the European Satellite Digital Multimedia Broadcast (S-DMB) Among Candidate Mobile Broadcast Solutions," IST Mobile \& Wireless Commun. Summit 2005, Dresden, Germany, June 19-23, 2005.

[5] C. Dovrolis et al., "Proportional Differentiated Services: Delay Differentiation and Packet Scheduling," IEEE Trans. Net., vol. 10, no. 1, Feb. 2002, pp. 12-26.

[6] M. Karaliopoulos et al., "Packet Scheduling for the Delivery of Multicast and Broadcast Services over SUMTS," Int'l. J. Satellite Commun. Net., vol. 22, 2004, pp. 503-32.

[7] 3GPP, TS 25.301 v6.2.0, "Radio Interface Protocol Architecture," May 2005

[8] S. Homer and A. Selman, Computability and Complexity Theory, Springer, 2000

[9] U. Mudugamuwa, "Reliable Multimedia Broadcast and Multicast Content Delivery Over 3G Mobile Satellite Networks," Ph.D. diss., School of Electronics and Physical Science, Univ. of Surrey, May 2006.

[10] 3GPP TS 23.107 V6.3.0, "Quality of Service (QoS) Concept and Architecture," June 2005.

\section{BIOGRAPHIES}

HONGFEI Du (H.Du@surrey.ac.uk) received his B.Eng. degree in electronic information engineering from the Department of Electronic Engineering, Beijing University of Aeronautics \& Astronautics, China, in 2003. He received his M.Sc. and M.Phil. degrees in mobile and satellite communications from the University of Surrey, United Kingdom, in 2004 and 2005, respectively. He is currently working toward a
Ph.D. degree at the Center for Communications Systems Research (CCSR) in the School of Electronic and Physical Science, University of Surrey. From 2004 to 2007 he was involved in several EU research projects in the area of mobile satellite communications systems and participated in the European Satellite Network of Excellence (SatNEx). His main research interests are in the area of mobile and satellite multimedia broadcasting, focusing on radio resource management, packet scheduling, quality of service support, and cross-layer design.

LINGHANG FAN (L.Fan@surrey.ac.uk) is a research fellow of the Mobile Communications Research Group at the University of Surrey. He received his B.Eng. in automatic control from Southeast University, China, and his M.Sc. and Ph.D. in telecommunications from the University of Bradford, United Kingdom. In 2003 he joined the University of Surrey and worked on the EU projects STRIKE, Ambient Networks, MAESTRO, and SatNEx. Currently, he is working on EU projects SATSIX and EC-GIN. His research interests include mobile communications, mobile Internet, and satellite communications. From 1998 to 2000 he was a researcher at the University of Bradford and worked on the EU projects SINUS and SUMO. He has published more than 20 papers in international journals and conferences.

UPENDRA MUdUgAMUWA (Upendra.Mudugamuwa@aeroflex. com) received a B.Eng. degree in electrical and electronic engineering from City University, London, United Kingdom, in 2001 . He received a Ph.D. degree in mobile and satellite communications from the University of Surrey in 2006 . He is currently working as a protocol software engineer at Aeroflex, Cambridge, United Kingdom. His main research interests are in the area of multimedia broadcast and multicast content delivery over $3 \mathrm{G}$ terrestrial and satellite networks, with emphasis on reliability of the content over radio access network, radio resource management, and cross-layer optimizations. During 2002 to 2006 he was involved in several EU research projects in the area of mobile satellite communications.

BARRY G. EvANs (B.Evans@surrey.ac.uk) received his B.Sc. and Ph.D. degrees in electrical engineering and microwave systems from the University of Leeds in 1965 and 1968, respectively. $\mathrm{He}$ is director of the Centre for Communications Systems Research at the University of Surrey in the United Kingdom, where he is a professor and also Pro-Vice Chancellor for Research and Enterprise. He is editor of the International Journal of Satellite Communications and Networking and a well-known international consultant, and has performed research for over 30 years in the field. He is the author of over 400 publications in the technical literature and of several books, including Satellite Communication Systems from IEE Press. Outside of the University of Surrey, he has been involved in several national and international committees, including the U.K. Foresight programs in communications and ITEC, EPSRC Strategic Advisory committees, and MoD-DSAC committees; as advisor to DG of OFTEL; board member of BNSC-TNAB; as well as ITU, ETSI, and the EU advisory committees. He is currently a member of the Ofcom Spectrum Advisory Board and the director of a small spin-off company, Mulsys Ltd. He has recently been appointed to the Steering Council of the European Technology Platform Integral Satcom Initiative and the HEFCE Strategic Advisory Committee for Business and Community. He was elected a Fellow of the Royal Academy of Engineering in 1991. 